POS $\quad \begin{aligned} & \text { PROCEEDINGS } \\ & \text { OF SCIENCE }\end{aligned}$

\title{
Top quark theory
}

\section{Peter Uwer*†}

Institut für Physik, Humboldt-Universität zu Berlin, D-10099 Berlin, Germany

E-mail: Peter.Uwer@Physik.HU-Berlin.de

Aspects of top-quark physics are reviewed with a special emphasis on recent theoretical developments.

XXth Hadron Collider Physics Symposium

November 16 - 20, 2009

Evian, France

\section{*Speaker.}

${ }^{\dagger}$ We acknowledge the support of the Initiative and Networking Fund of the Helmholtz Association, contract HA-101 ("Physics at the Terascale"). 


\section{Introduction}

The top quark with a mass of $173.1 \pm 0.6 \pm 1.1 \mathrm{GeV} / c^{2}[1]$ is the heaviest fermion discovered so far. Owing to its large mass the top quark has remarkable properties. In the context of quantum chromodynamics - the accepted theory of strong interaction — the top quark is the only quark which does not produce bound states. Due to the large mass the width is so large that on average the top quark decays before it can hadronize [2]. The spin information of the top quark-a footprint of the production mechanism - is thus not diluted by hadronisation but transferred to the decay products. In the Standard Model (SM) where the dominant decay is the parity-violating decay into a $W$-boson and a $b$-quark the polarisation can be analysed via the angular distribution of the decay products. The polarisation of the top-quark can thus be used as an additional observable for more precise tests of the underlying interaction. Since the mass of the top quark is so large top-quark physics will probe nature at the energy scale $\sim 170 \mathrm{GeV}$-close to the scale where electroweak symmetry breaking takes place. It is thus natural to use top-quark physics as a tool to explore this energy regime. This is of particular interest since the fact that the top-quark mass is so close to the scale of electroweak symmetry breaking has motivated many extensions of the SM in which the top-quark places a central role. It is thus important to measure as precisely as possible the properties of the top-quark and to compare these measurements with the SM predictions to see whether the top-quark behaves as predicted by the SM or whether new physics effects become visible. In the SM the topquark properties are fixed by the gauge structure. The top quark is predicted to be an object with electric charge $2 / 3$ and the normal up-type quark couplings to the massive gauge bosons. The only free parameters in the SM are the top-quark mass or alternatively its Yukawa coupling to the Higgs boson and the matrix elements $V_{t d}, V_{t s}, V_{t b}$ of the Cabibbo-Kobayashi-Maskawa matrix. Assuming unitarity and 3 families these elements are highly constrained from indirect measurements. It turns out that $\left|V_{t b}\right|$ is close to one while the remaining two are close to zero. The dominant decay in the $\mathrm{SM}$ is thus the aforementioned decay $t \rightarrow W b$. Since the bounds on $V_{t b}$ are very much relaxed if a fourth family is taken into account it is important to extract the value $V_{t b}$ from a direct measurements in single-top production. This has been done recently at the Tevatron [] and will be continued at the LHC. The top-quark mass is also known very precisely from the measurements at Tevatron. The current experimental uncertainty is less than $1 \%$. However one should keep in mind that the interpretation of the measured quantity in terms of a parameter of the SM Lagrangian in a specific renormalisation scheme is still not fully settled. It is evident that for precise experimental studies equally precise theoretical predictions are required to compare with. Since the large width of the top-quark effectively cuts off non-perturbative QCD effects reliable predictions are feasible within QCD allowing also to include corrections beyond the Born approximation. A central quantity in that context is the total cross section for top-quark pair production. We will comment on recent theoretical progress concerning the total cross section in section 2. QCD can lead to a chargeasymmetry in top-quark pair production. We will briefly comment on this observable where a significant deviation from the theory prediction is observed in section 3. In section 4 the first determination of the running top-quark mass at a high scale is discussed. Some recent progress in top-quark physics which cannot be discussed in detail will be quoted briefly in section 5 . 


\section{Recent theoretical progress in top-quark pair production}

Top-quark pair production in hadronic collisions proceeds in Born approximation via the parton channels $g g \rightarrow t \bar{t}$ and $q \bar{q} \rightarrow t \bar{t}$. The hadronic cross section is obtained from a convolution of the partonic cross sections with the parton distribution functions (pdf). Due to the very different collider energy at which the pdf's are probed the cross section at LHC is dominated by gluon fusion while at the Tevatron the quark channel dominates. The next-to-leading order corrections were calculated 20 years ago for unobserved spins in Ref. $[3,4]$ and with the full top-quark spin dependence in Ref. [5, 6]. As usual the corrections are a combination of virtual and real corrections. Due to the complicated phase integrals involved the results were only quoted as fits to numerically integrated cross sections. Only recently a complete analytic result for the NLO partonic cross section has been published [7]. The behaviour close to the production threshold however was already known analytically in the early work [3]. For the gluon channel the threshold behaviour reads in NLO:

$$
\left.\delta \sigma_{g g}^{\mathrm{NLO}}\right|_{\text {threshold }} \sim \frac{11}{42} \frac{\pi^{2}}{\beta}+12 \ln ^{2}\left(8 \beta^{2}\right)-\frac{366}{7} \ln \left(8 \beta^{2}\right), \quad \beta=\sqrt{1-\frac{4 m_{t}^{2}}{s}} .
$$

One can observe the enhancement close to the threshold due to the $1 / \beta$ Coulomb singularity and the soft logarithms $\ln ^{2}(\beta), \ln (\beta)$. A similar structure can be found in the quark channel. Close to the threshold both contributions can give potentially large corrections spoiling the validity of the fixed order calculation. Owing to the universal properties of the specific corrections the resummation to all orders in perturbation theory is possible. For the soft-logarithms this has been done up to the next-to-leading log (NLL) level for example in Refs. [8, 9]. In Ref. [10] this analysis has been extended to the next-to-next-to-leading (NNLL) order based on known existing results and a physically motivated guess for the missing piece $D_{Q Q}$. The NNLL resummation gives only a minor correction compared to NLL. Recently $D_{Q Q}$ has been reanalysed and a value differing from Ref.[10] has been found $[11,12,13]$, however the phenomenological conclusions drawn in Ref. [10] remain unchanged. Recently also much progress has been made concerning the calculation of the next-to-next-to-leading order (NNLO) corrections for the inclusive cross section. This includes a better understanding of the factorization of scattering amplitudes for massive quarks $[14,13]$ but also results for the two-loop amplitudes $[15,16,17]$ and the one-loop amplitudes

squared $[18,19,20]$. The combination of the various corrections in particular the cancellation of soft and collinear singularities between double bremsstrahlung corrections and the two-loop contribution still needs to be done. One may wonder whether one can improve the quality of the theoretical predictions already in the meantime taking the theoretical progress into account. This has been studied in Ref. [10]. The basic idea is to use the knowledge about the universal behaviour due to soft gluon resummation to derive an approximation to the full NNLO result (see also Ref. [21]). In Refs. $[10,22]$ the NNLL resummation was used to predict the threshold corrections in NNLO. This ansatz is further improved by adding Coulomb type corrections and the known scale dependent (factorization as well as renormalization scale) terms. Since corrections due to the emission of hard jets are small $[23,24]$ one can expect that the approximation based on soft gluon approximation which gives the correct behaviour in the threshold region should provide a good approximation to NNLO. This is in particular true if the production is threshold dominated. For the CTEQ6.6 pdf set the results quoted in Ref. [22] are $\sigma_{\mathrm{LHC}}=874_{-33}^{+9}(\text { scale })_{-28}^{+28}(\mathrm{CTEQ6.6}) \mathrm{pb}$, 
$\sigma_{\mathrm{Tev}}=7.34_{-0.28}^{+0.24}(\text { scale })_{-0.41}^{+0.41}($ CTEQ6.6) $\mathrm{pb}$. As far as the central values are concerned similar results have been obtained recently in Refs. [25, 26]. However the uncertainty due to the scale dependence quoted in Ref. [10] is much smaller due to the inclusion of all scale dependent terms. One should keep in mind that the resummation used in Ref. [25] also generates scale dependent parts at the NNLO level. The difference compared to Ref. [10] is that these terms are only generated for the soft part. How to estimate the theoretical uncertainties of the various theoretical predictions is currently under investigation. In Eq.(2.1) the cross section may also be enhanced due to the presence of the Coulomb type corrections. This is a remnant of a would be bound state. The corrections can be resummed using a non-relativistic Green function [27, 28]. Similar to what is known from $e^{+} e^{-}$annihilation a peak structure in the pair invariant mass $m_{t \bar{t}}$ distribution is observed below the nominal threshold [27, 28]. If this structure is resolved at the LHC it provides a sensitive measure to the top-quark mass. The region below the threshold where the fixed order cross section is zero gives a correction to the inclusive cross section of about 10pb. If the cross section is measured with a 5\% accuracy these corrections as well as the weak corrections $[29,30,31]$ must be included in the theoretical predictions.

\section{The forward-backward charge asymmetry}

Radiative corrections to top-quark pair production can lead to interference terms in the cross section which are odd under charge conjugation of the top quarks. This effect has been studied in Refs. [32, 33]. At the Tevatron the charge asymmetry can lead to a forward-backward asymmetry $A_{f b}$ of the top quarks. In Ref. [34] the effect is predicted to be $A_{f b}=0.05 \pm 0.015$. The most recent measurement from the CDF collaboration based on the integrated luminosity of $3.21 / \mathrm{fb}$ finds a significant difference: $A_{f b}^{\text {exp. }}=0.193 \pm 0.065^{\text {stat. }} \pm 0.024^{\text {syst. }}$. The interpretation of the discrepancy is unclear. Despite the fact that in Refs. [32, 33] one-loop diagrams and real corrections were calculated the prediction is only at leading-order accuracy since the asymmetry appears for the first time at this order. Higher-order corrections could shift the theoretical prediction towards the experimental value. For jet production in association with an additional jet the situation is different. Here the asymmetry appears already in the Born approximation. The NLO corrections for that process calculated in Refs. [23, 24] allows to calculate also corrections for the asymmetry. This has been studied in Refs. $[23,24]$ using different values for $p_{T}^{\text {cut }}$ used to define the additional jet. The main result is that the asymmetry receives large radiative corrections and is almost washed out at NLO. The origin of this behaviour and the corresponding behaviour of the observable defined for the inclusive sample needs to be clarified. A possible explanation could also be the presence of new physics (see for example [34, 35, 36, 37]).

\section{A direct determination of the running top-quark mass}

The on-shell mass of a quark is not a well defined concept due to intrinsic uncertainties of the order of $\Lambda_{\mathrm{QCD}}[38,39]$. Furthermore the experimental analysis at the Tevatron is mostly based on a leading-order prescription which is insufficient to measure a parameter of the Lagrangian. An alternative method addressing both aspects is proposed in Ref. [22]. The idea is to use the sensitivity of the total cross section to the top-quark mass to estimate the parameter from the measured cross 
section. Converting the theoretical predictions to the running mass ( $\overline{\mathrm{MS}}$ scheme) gives a direct handle to the running mass at large scale. This has been done for the first time in Ref. [22]. As a remarkable result it is observed that the perturbative prediction becomes much more stable with respect to radiative corrections leading to a very stable determination of the running mass. The value quoted in Ref. [22] is $160_{-3.2}^{+3.3} \mathrm{GeV} / c^{2}$. The same procedure applied in the on-shell scheme gives rather different results when applied in LO, NLO, and NNLO. Converting the running mass to the on-shell mass the result is consistent with the direct measurements. Due to the weak sensitivity of the cross section with respect to the mass the method is not competitive with the direct measurements as far as the uncertainty is concerned, however the method provides an independent cross check and is theoretically rather clean.

\section{Recent theoretical progress}

In the following we briefly list recent theoretical progress in top quark physics:

- QCD corrections to top-quark pair production with an additional jet: In Refs. [23, 24] the NLO QCD corrections are presented. The corrections are of the order of 10-20\% however also the shapes of some distributions are modified.

- Single-top quark production in the 4-flavour scheme: In Ref. [40, 41] single top-quark production is studied in the 4-flavour scheme. While for inclusive quantities the results are comparable to the 5-flavour scheme differences are observed in distributions sensitive to the spectator b-quark.

- QCD corrections to $t \bar{t} b \bar{b}$ production: This process is a background for Higgs studies in the $t \bar{t} H$ finale state. Since the calculation involve one-loop corrections to six-point amplitudes the calculation is highly non-trivial. NLO results have been presented recently in Refs. [42, 43, 44].

- Top mass effects in Higgs production via gluon fusion: Higgs production via gluon fusion proceeds through a top-quark loop. Higher order corrections are typically calculated replacing this loop with an effective vertex. In Ref. [45, 46] mass corrections to this approach were studied in NNLO. The corrections are small and proof the applicability of the effective vertex approximation for the relevant cases.

Acknowledgments: I would like to thank the organisers for the invitation to this conference which I enjoyed a lot. My apologies that not all recent developments could have been mentioned due to the lack of time.

\section{References}

[1] Tevatron Electroweak Working Group, (2009), 0903.2503,

[2] I.I.Y. Bigi et al., Phys. Lett. B181 (1986) 157,

[3] P. Nason, S. Dawson and R.K. Ellis, Nucl. Phys. B303 (1988) 607,

[4] W. Beenakker et al., Phys. Rev. D40 (1989) 54,

[5] W. Bernreuther et al., Phys. Rev. Lett. 87 (2001) 242002, hep-ph/0107086,

[6] W. Bernreuther et al., Nucl. Phys. B690 (2004) 81, hep-ph/0403035,

[7] M. Czakon and A. Mitov, Nucl. Phys. B824 (2010) 111, 0811.4119,

[8] R. Bonciani et al., Nucl. Phys. B529 (1998) 424, hep-ph/9801375,

[9] N. Kidonakis and G. Sterman, Nucl. Phys. B505 (1997) 321, hep-ph/9705234, 
[10] S. Moch and P. Uwer, Phys. Rev. D78 (2008) 034003, 0804.1476,

[11] M. Czakon, A. Mitov and G. Sterman, Phys. Rev. D80 (2009) 074017, 0907.1790,

[12] M. Beneke, P. Falgari and C. Schwinn, Nucl. Phys. B828 (2010) 69, 0907.1443,

[13] A. Ferroglia et al., JHEP 11 (2009) 062, 0908.3676,

[14] A. Mitov and S. Moch, JHEP 05 (2007) 001, hep-ph/0612149,

[15] M. Czakon, A. Mitov and S. Moch, Phys. Lett. B651 (2007) 147, 0705.1975,

[16] M. Czakon, A. Mitov and S. Moch, Nucl. Phys. B798 (2008) 210, 0707.4139,

[17] R. Bonciani et al., JHEP 08 (2009) 067, 0906.3671,

[18] J.G. Korner, Z. Merebashvili and M. Rogal, Phys. Rev. D77 (2008) 094011, 0802.0106,

[19] B. Kniehl et al., Phys. Rev. D78 (2008) 094013, 0809.3980,

[20] C. Anastasiou and S.M. Aybat, Phys. Rev. D78 (2008) 114006, 0809.1355,

[21] M. Beneke et al., (2009), 0911.5166,

[22] U. Langenfeld, S. Moch and P. Uwer, Phys. Rev. D80 (2009) 054009, 0906.5273,

[23] S. Dittmaier, P. Uwer and S. Weinzierl, Phys. Rev. Lett. 98 (2007) 262002, hep-ph/0703120,

[24] S. Dittmaier, P. Uwer and S. Weinzierl, Eur. Phys. J. C59 (2009) 625, 0810.0452,

[25] M. Cacciari et al., JHEP 09 (2008) 127, 0804.2800,

[26] N. Kidonakis and R. Vogt, Phys. Rev. D78 (2008) 074005, 0805.3844,

[27] K. Hagiwara, Y. Sumino and H. Yokoya, Phys. Lett. B666 (2008) 71, 0804.1014,

[28] Y. Kiyo et al., Eur. Phys. J. C60 (2009) 375, 0812.0919 ,

[29] W. Beenakker et al., Nucl. Phys. B411 (1994) 343,

[30] J.H. Kuhn, A. Scharf and P. Uwer, Eur. Phys. J. C51 (2007) 37, hep-ph/0610335,

[31] W. Bernreuther, M. Fucker and Z.G. Si, Phys. Rev. D78 (2008) 017503, 0804.1237,

[32] J.H. Kuhn and G. Rodrigo, Phys. Rev. Lett. 81 (1998) 49, hep-ph/9802268,

[33] J.H. Kuhn and G. Rodrigo, Phys. Rev. D59 (1999) 054017, hep-ph/9807420,

[34] O. Antunano, J.H. Kuhn and G. Rodrigo, Phys. Rev. D77 (2008) 014003, 0709.1652,

[35] P.H. Frampton, J. Shu and K. Wang, (2009), 0911.2955,

[36] C.P. Burgess, M. Trott and S. Zuberi, JHEP 09 (2009) 082, 0907.2696,

[37] A. Djouadi et al., (2009), 0906.0604,

[38] I.I.Y. Bigi et al., Phys. Rev. D50 (1994) 2234, hep-ph/9402360,

[39] M. Beneke and V.M. Braun, Nucl. Phys. B426 (1994) 301, hep-ph/9402364,

[40] J.M. Campbell et al., Phys. Rev. Lett. 102 (2009) 182003, 0903.0005,

[41] J.M. Campbell et al., JHEP 10 (2009) 042, 0907.3933,

[42] A. Bredenstein et al., JHEP 08 (2008) 108, 0807.1248,

[43] A. Bredenstein et al., Phys. Rev. Lett. 103 (2009) 012002, 0905.0110,

[44] G. Bevilacqua et al., JHEP 09 (2009) 109, 0907.4723,

[45] R.V. Harlander and K.J. Ozeren, JHEP 11 (2009) 088, 0909.3420,

[46] A. Pak, M. Rogal and M. Steinhauser, (2009), 0911.4662, 